\title{
Second-Order Containment Control of Multiagent Systems in the Presence of Uncertain Topologies with Time-Varying Delays
}

\author{
Fuyong Wang, ${ }^{1,2}$ Hongyong Yang, ${ }^{3}$ Zhongxin Liu, ${ }^{1,2}$ and Zengqiang Chen ${ }^{1,2}$ \\ ${ }^{1}$ College of Computer and Control Engineering, Nankai University, Tianjin 300353, China \\ ${ }^{2}$ Tianjin Key Laboratory of Intelligent Robotics, Nankai University, Tianjin 300353, China \\ ${ }^{3}$ College of Information and Electrical Engineering, Ludong University, Yantai 264025, China \\ Correspondence should be addressed to Zhongxin Liu; lzhx@nankai.edu.cn
}

Received 30 September 2016; Revised 1 December 2016; Accepted 18 December 2016; Published 10 January 2017

Academic Editor: Xian Zhang

Copyright (C) 2017 Fuyong Wang et al. This is an open access article distributed under the Creative Commons Attribution License, which permits unrestricted use, distribution, and reproduction in any medium, provided the original work is properly cited.

\begin{abstract}
This paper considers the containment control problem of second-order multiagent systems in the presence of time-varying delays and uncertainties with dynamically switching communication topologies. Moreover, the control algorithm is proposed for containment control, and the stability of the proposed containment control algorithm is studied with the aid of Lyapunov-Krasovskii function when the communication topology is jointly connected. Some sufficient conditions in terms of linear matrix inequalities (LMIs) are provided for second-order containment control with multiple stationary leaders. Finally, simulations are given to verify the effectiveness of the obtained theoretical results.
\end{abstract}

\section{Introduction}

Recently, there has been a growing interest towards development of the distributed cooperative control of multiagent systems (MASs). To our knowledge, plenty of theoretical results about consensus [1-5] and containment control [69] in distributed cooperative control of MASs have been obtained. However, in practical applications, due to communication delays and uncertain topologies that always emerge, analysis and synthesis of distributed cooperative problem have become more complex and difficult. Meanwhile, it is practically significant to investigate the containment control of MASs with delays and uncertainties.

For consensus problems of MASs with uncertain topologies, the average consensus with time-varying communication delays is investigated in [10], and several sufficient conditions for average consensus are derived in terms of linear matrix inequalities (LMIs). In [11], the robust discretetime consensus problem of MASs with uncertain topologies is addressed, and a necessary and sufficient condition for robust discrete-time consensus is obtained by the Lyapunov stability theory. Considering both fixed and switching directed topologies, the average consensus problem in MASs with uncertain topologies and multiple time-varying delays is studied in [12]. For linear time-invariant MASs over Markovian switching networks, stochastic consensus problems with time-varying delays and uncertain topologies are analyzed in [13]. In [14], the consensus problem of MASs in the presence of uncertain topologies with time-varying delays is analyzed by a new approach, and a condition in terms of linear matrix inequalities is presented for consensus for MASs with switching topologies.

As a kind of extended consensus problem, containment control has been paid much attention, which aims to design appropriate control protocols to drive the followers to a target area (convex hull formed by the leaders) asymptotically. For linear MASs, the cooperative containment control problem is discussed in [15], and several necessary and sufficient containment conditions are presented by using spectral analysis and matrix theory. Considering unconnected topologies of MASs, the containment control for both first-order and second-order MASs with jointly connected topologies is studied in [16]. Considering communication delays, containment control for second-order MASs with time-varying delays is studied in [17]; both the case with multiple dynamic leaders and the case with multiple stationary leaders are discussed. In [18], the containment control problem for secondorder MASs with time-varying delays and jointly connected 
topologies is investigated, and the stability of the proposed control algorithm is analyzed by Lyapunov-Krasovskii method. For uncertain linear MASs, the containment control problem with the dynamic agents described by fractionalorder differential equations is investigated in [19], and some sufficient conditions are presented by the stability theory of fractional-order systems and matrix theory. The abovementioned works implicitly assume the link weights or interaction strengths can be exactly measured; however, environmental uncertainties and measurement error cannot be ignored in real systems. Motivated by these considerations, the containment control of first-order MASs with uncertain topologies and communication time delays is studied in [20], and the sufficient condition for containment control of MASs under jointly connected topologies is derived with the aid of Lyapunov-Krasovskii function.

Two important factors always emerge in some real situations: one is that some uncertainties may exist in the MASs due to measurement error and environmental uncertainties cannot be avoided in real systems and another is that time delays are usually inevitable because of the possible slow process of interactions among the agents in communication networks. Motivated by these two factors, lots of distributed consensus protocols have been developed by some researchers for MASs with uncertainties and delayed communications. However, different from most of those current literatures, for the MASs with uncertainties and time-varying delays, we aim to analyze and investigate the distributed containment control problem with multiple leaders and jointly connected topologies. The algorithms proposed here can ensure that the uncertain MASs achieve containment control in the presence of switching topologies with multiple stationary leaders. By applying linear matrix inequality method, the convergence of the algorithm for the MASs with dynamically switching topologies is analyzed by Lyapunov-Krasovskii method. Finally, numerical simulations are provided to illustrate the effectiveness of the conclusion in this paper.

The paper is organized as follows. In Section 2, some basic concepts in algebraic graph theory and some related lemmas are presented. In Section 3, the main results for second-order MASs with uncertain topologies and time-varying delays are obtained. Then the containment control protocol is presented for MASs with multiple stationary leaders and dynamically switching topologies. Finally, numerical simulations and conclusion are given in Sections 4 and 5, respectively.

\section{Preliminaries}

Let $G=(W, E, A)$ be an undirected graph consisting of a note set $W=\{1,2, \ldots, n\}$, an edge set $E \in W \times W$, and a weighted adjacency matrix $A=\left[a_{i j}\right] \in \mathbb{R}^{n \times n}$. The elements of $A$ satisfy $a_{i j}>0$ if $(j, i) \in E, a_{i j}=0$; otherwise, where $(j, i)$ represents an edge of $G,\{j, i\} \in W$. Here, we assume that $(i, i) \notin E$; that is, $a_{i i}=0$. Let $N_{i}=\{j \in W \mid(j, i) \in E, j \neq i\}$ denote the set of the neighbors of node $i$. The Laplacian matrix corresponding to the undirected graph $G$ is defined as $L=\left[l_{i j}\right] \in \mathbb{R}^{n \times n}$, where $l_{i j}=-a_{i j}, j \neq i$, and $l_{i j}=\sum_{j \in N_{i}} a_{i j}, j=i$.
A path between two distinct notes $i$ and $j$ is a finite ordered sequence of distinct edges of $G$ with the form $\left(i_{1}, i_{2}\right),\left(i_{2}, i_{3}\right), \ldots,\left(i_{\pi-1}, i_{\pi}\right)$, where $i_{1}=i$ and $i_{\pi}=j$. The undirected graph $G$ is said to be connected if there is a path between any distinct pair of nodes. The union of a collection of graphs $G_{1}, G_{2}, \ldots, G_{q}$ with the same note set $W$ is defined as the graph $G_{1-q}$ with the note set $W$ and edge set equal to the union of the edge sets of all of the graphs in the collection. Moreover, $G_{1}, G_{2}, \ldots, G_{q}$ is jointly connected if its union graph $G_{1-q}$ is connected [21].

Consider an infinite sequence of nonempty, bounded, and contiguous time intervals $\left[t_{r}, t_{r+1}\right), r=1,2, \ldots$, with $t_{1}=0$ and $t_{r+1}-t_{r} \leq T_{a}, T_{a}>0$. In each interval $\left[t_{r}, t_{r+1}\right)$, there is a sequence of subintervals $\left[t_{r, s}, t_{r, s+1}\right)$ with $t_{r, 1}=t_{r}$ and $t_{r, m_{r}+1}=t_{r+1}$ satisfying $t_{r, s+1}-t_{r, s} \geq T_{b}$, where $T_{b}>0$ and $s=1,2, \ldots, m_{r}, m_{r} \geq 1$. The communication topology described by $G_{r, s}$ switches at $t_{r, s}$ and it does not change during each subinterval $\left[t_{r, s}, t_{r, s+1}\right)$. Suppose that the MASs represented by the communication topology graph $G$ consist of $n$ followers and $m$ leaders. Moreover, when the topology of MASs is dynamically switching, we introduce a piecewise constant switching function $\sigma(t):[0,+\infty) \rightarrow P$, $P=\{1,2, \ldots, p\}, \sigma=\sigma(t), \sigma \in P$, where $p$ denotes the total number of all possible communication topologies. Then the communication topology of MASs described by $G_{\sigma}$ switches at $t$ and its Laplacian matrix is denoted by $L_{\sigma}$, and the communication topology among the $n$ followers described by $G_{\sigma F}$ switches at $t$ and its Laplacian matrix is denoted by $L_{\sigma F}$.

In MASs, an agent is called a leader if it has no neighbor or a follower if it has at least one neighbor [22]. Considering a MAS with $n$ followers and $m$ leaders, since the leaders have no neighbors, the Laplacian matrix $L$ associated with the communication graph $G$ can be partitioned as

$$
L=\left[\begin{array}{cc}
L_{F} & L_{F \Re} \\
0_{m \times n} & 0_{m \times m}
\end{array}\right],
$$

where $L_{F} \in \mathbb{R}^{n \times n}$ and $L_{F \Re} \in \mathbb{R}^{n \times m}$. The following lemma about $L_{F}$ and $L_{F \Re}$ is brought in, which is useful for deriving the main results.

Lemma 1 (see [23]). If there exists at least a path to a leader for each follower, then $L_{F}$ in (1) is positive definite, $-L_{F}^{-1} L_{F \Re}$ is a nonnegative matrix, and the sum of the entries in each row equals 1 .

Definition 2 (see [24]). Let $X=\left\{\xi_{1}, \xi_{2}, \ldots, \xi_{n}\right\}$ be a set in a real vector space $Z \subseteq \mathbb{R} . \operatorname{CO}(X)=\left\{\sum_{i=1}^{n} \beta_{i} \xi_{i} \mid \xi_{i} \in X, \beta_{i} \in\right.$ $\left.\mathbb{R}, \beta_{i} \geq 0, \sum_{i=1}^{n} \beta_{i}=1\right\}$ denotes the convex hull of the set $X$.

Lemma 3 (see [25]). For any real differentiable vector function $z(t) \in \mathbb{R}^{n}$ and any $n \times n$ dimensional constant matrix $M=$ $M^{\mathrm{T}}>0$, we have the following inequality:

$$
\begin{aligned}
& {[z(t)-z(t-\tau(t))]^{\mathrm{T}} M[z(t)-z(t-\tau(t))]} \\
& \quad \leq h \int_{t-\tau(t)}^{t} \dot{z}^{\mathrm{T}}(s) M \dot{z}(s) d s
\end{aligned}
$$

where $t \geq 0,0 \leq \tau(t) \leq h$. 
Lemma 4 (see [26]). Let $Q=Q^{T}, F_{1}, F_{2}$, and $S(t)$ be matrixes with appropriate dimensions, and matrix $S(t)$ satisfies $S^{\mathrm{T}}(t) S(t) \leq I$. Then

$$
Q+F_{1} S(t) F_{2}+F_{2}^{\mathrm{T}} S^{\mathrm{T}}(t) F_{1}^{\mathrm{T}}<0,
$$

holds if and only if there exists a positive constant $\varepsilon>0$ such that

$$
Q+\varepsilon^{-1} F_{1} F_{1}^{\mathrm{T}}+\varepsilon F_{2}^{\mathrm{T}} F_{2}<0 .
$$

Lemma 5 (see [27]). Let $\phi(t): \mathbb{R} \geq 0 \rightarrow \mathbb{R}$ be a piecewise continuous function with the following properties:

(1) $\phi(t)$ is continuous and differentiable on each subinterval $\left[t_{r, s}, t_{r, s+1}\right)$ and switches at $t_{r, s}, r=1,2, \ldots$, $s=1,2, \ldots, m_{r}$. Moreover, $\phi(t)$ is right continuous and right differentiable function at $t_{r, s}$.

(2) The derivative (including the right derivative) of $\phi(t)$ for any $t \in[0,+\infty)$ is bounded; that is, $|\dot{\phi}(t)| \leq \omega$ for any $t \in[0,+\infty)$ and some constant $\omega>0$.

(3) $\lim _{t \rightarrow+\infty} \int_{0}^{t} \phi(\tau) d \tau$ exists and is finite.

Then $\lim _{t \rightarrow+\infty} \phi(t)=0$.

\section{Main Results}

Consider second-order MASs consisting of $n$ followers and $m$ stationary leaders. The followers' set and the leaders' set are denoted by $F=\{1,2, \ldots, n\}$ and $\Re=\{n+1, n+2, \ldots, n+m\}$, respectively. The dynamics of each agent can be described by the following equation:

$$
\begin{aligned}
& \dot{q}_{i}(t)=p_{i}(t), \\
& \dot{p}_{i}(t)=u_{i}(t), \quad i \in\{F, \Re\},
\end{aligned}
$$

where $q_{i}(t) \in \mathbb{R}^{N}, p_{i}(t) \in \mathbb{R}^{N}$, and $u_{i}(t) \in \mathbb{R}^{N}$ are the position vector, the velocity vector, and the control input vector of the $i$ th agent, respectively. For simplicity, we assume that $N=1$ in this paper and the case of $N>1$ can be obtained with the Kronecker product.

In a network with uncertain topology, we consider the following containment control algorithm for (5):

$$
\begin{aligned}
& p_{i}(t)=0, \quad i \in \Re ; \\
& u_{i}(t) \\
& =-k_{1} p_{i}(t) \\
& \quad-\sum_{j \in N_{i}}\left(a_{i j}+\Delta a_{i j}(t)\right)\left(q_{i}(t-\tau(t))-q_{j}(t-\tau(t))\right),
\end{aligned}
$$$$
i \in F \text {, }
$$

where $k_{1}>0$ is the feedback gain, $\Delta a_{i j}(t)$ and $\Delta a_{i i}(t)=0$ are uncertainties, and $\tau(t)$ is the time-varying communication delay. Here the uncertainties $\Delta a_{i j}(t)$ exist in the control algorithm, since measurement error and environmental uncertainties cannot be avoided in real systems.
Assumption 6. The time-varying communication delay $\tau(t)$ in (6) is bounded; that is, $0 \leq \tau(t)<h$, where $h>0, t \geq 0$.

Assumption 7. In each interval $\left[t_{r}, t_{r+1}\right), r=1,2, \ldots$, the communication topologies of MASs are jointly connected.

Definition 8. $\Delta L(t)=\left[\Delta l_{i j}(t)\right]$ is the uncertain matrix of MASs, which is defined as $\Delta l_{i j}(t)=\sum_{j \in N_{i}} \Delta a_{i j}(t), i=j$, and $\Delta l_{i j}(t)=-\Delta a_{i j}(t), i \neq j$. For the MASs with $n$ followers and $m$ leaders, the uncertain matrix $\Delta L(t)$ can be partitioned into $\Delta L(t)=\left[\begin{array}{cc}\Delta L_{F}(t) & \Delta L_{\mathscr{F}}(t) \\ 0_{m \times n} & 0_{m \times m}\end{array}\right]$, where $\Delta L_{F}(t) \in \mathbb{R}^{n \times n}, \Delta L_{F \Re}(t) \epsilon$ $\mathbb{R}^{n \times m}$.

In this paper, we assume that the norm bounded parameter uncertainty satisfies

$$
\begin{aligned}
\Delta L(t) & =C_{1} S(t) C_{2} \\
\Delta L_{F}(t) & =D_{1} S(t) D_{2},
\end{aligned}
$$

where $C_{1}, C_{2}, D_{1}$, and $D_{2}$ are constant matrixes with appropriate dimensions, and the diagonal matrix $S(t)$ satisfies

$$
S^{\mathrm{T}}(t) S(t) \leq I .
$$

It follows that the uncertainties of MASs satisfy

$$
\begin{aligned}
\Delta L^{\mathrm{T}}(t) \Delta L(t) & \leq \alpha^{2} I_{n+m} \\
\Delta L_{F}^{\mathrm{T}}(t) \Delta L_{F}(t) & \leq \alpha^{2} I_{n},
\end{aligned}
$$

where $\alpha>0$ is an appropriate constant.

Let $x_{F}(t)=\left[q_{F}^{\mathrm{T}}(t), p_{F}^{\mathrm{T}}(t)\right]^{\mathrm{T}}$ and $x_{L}(t)=\left[q_{L}^{\mathrm{T}}(t), p_{L}^{\mathrm{T}}(t)\right]^{\mathrm{T}}$, where $q_{F}(t)=\left[q_{1}(t), q_{2}(t), \ldots, q_{n}(t)\right]^{\mathrm{T}}, p_{F}(t)=\left[p_{1}(t)\right.$, $\left.p_{2}(t), \ldots, p_{n}(t)\right]^{\mathrm{T}}, q_{L}(t)=\left[q_{n+1}(t), q_{n+2}(t), \ldots, q_{n+m}(t)\right]^{\mathrm{T}}$, and $p_{L}(t)=\left[p_{n+1}(t), p_{n+2}(t), \ldots, p_{n+m}(t)\right]^{\mathrm{T}}$. By Definition 8, it follows that the dynamics of MASs resulting from (5) to (6) can be written in a matrix form as

$$
\begin{aligned}
& \dot{x}_{L}(t)=H_{1} x_{L}(t) \\
& \dot{x}_{F}(t)=-H_{2} x_{F}(t-\tau(t))-H_{3} x_{L}(t-\tau(t)),
\end{aligned}
$$

where $H_{1}=\left[\begin{array}{cc}0_{m \times m} & I_{m \times m} \\ 0_{m \times m} & 0_{m \times m}\end{array}\right], H_{2}=\left[\begin{array}{cc}0_{n \times n} & -I_{n} \\ E_{F} & k_{1} I_{n}\end{array}\right]$, and $H_{3}=$ $\left[\begin{array}{cc}0_{n \times m} & 0_{n \times m} \\ E_{F \Re} & 0_{n \times m}\end{array}\right], E_{F}=L_{F}+\Delta L_{F}(t), E_{F \Re}=L_{F \Re}+\Delta L_{F \Re}(t)$. Since there are multiple stationary leaders in MASs, that is, $p_{L}(t)=p_{L}(0)=0$, we get $\dot{x}_{L}(t)=H_{1} x_{L}(t)=\left[\begin{array}{c}p_{L}(t) \\ 0\end{array}\right]=0$ in (12).

Let $\tilde{x}_{F}(t)=x_{F}(t)+H_{2}{ }^{-1} H_{3} x_{L}(t)$; then (12) can be transformed into the following form:

$$
\dot{\tilde{x}}_{F}(t)=-H_{2} \widetilde{x}_{F}(t-\tau(t)),
$$

where $H_{2}^{-1}=\left[\begin{array}{ccc}k_{1} E_{F}^{-1} & E_{F}^{-1} \\ -I_{n} & 0_{n \times n}\end{array}\right]$.

Lemma 9. Let $x_{L}{ }^{*}=\lim _{t \rightarrow \infty} x_{L}(t)$ and $x_{F}{ }^{*}=\lim _{t \rightarrow \infty} x_{F}(t)$. The containment control of the second-order MASs can be achieved if $x_{F}{ }^{*} \rightarrow-H_{2}{ }^{-1} H_{3} x_{L}{ }^{*}$. 
Proof. By Lemma 1, it follows that $-E_{F}^{-1} E_{F \Re}$ is a nonnegative matrix and the sum of the entries in each row equals 1. Furthermore, $-E_{F}^{-1} E_{F \Re} q_{L}{ }^{*}$ is located in the convex hull formed by the set of $q_{L}{ }^{*}$ from Definition 2, where $q_{L}{ }^{*}=$ $\lim _{t \rightarrow \infty} q_{L}(t)$. Thus, if $x_{F}{ }^{*} \rightarrow-H_{2}{ }^{-1} H_{3} x_{L}{ }^{*}$, that is, $\left(\begin{array}{c}q_{F}{ }^{*} \\ p_{F}{ }^{*}\end{array}\right) \rightarrow$

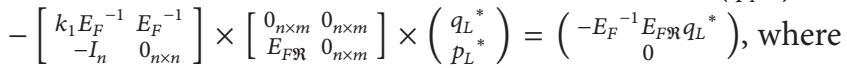
$q_{F}{ }^{*}=\lim _{t \rightarrow \infty} q_{F}(t), p_{F}{ }^{*}=\lim _{t \rightarrow \infty} p_{F}(t)$, and $p_{L}{ }^{*}=p_{L}(0)=$ 0 , then the containment control of second-order MASs can be achieved.

Suppose the communication graph $G_{\sigma}$ on subinterval $\left[t_{r, s}, t_{r, s+1}\right)$ has $n_{\sigma} \geq 1$ connected subgraphs $G_{\sigma}^{i}$, and each connected subgraph $G_{\sigma}^{i}$ has $d_{\sigma}^{i}=d_{\sigma F}^{i}+d_{\sigma \Re}^{i}$ nodes, where $d_{\sigma F}^{i} \geq 1$ represents the number of followers, $d_{\sigma \Re}^{i} \geq 1$ represents the number of leaders, $i=1,2, \ldots, n_{\sigma}$. The Laplacian matrix of subgraph $G_{\sigma}^{i}$ is denoted by $L_{\sigma}^{i}$, and the uncertain matrix of subgraph $G_{\sigma}^{i}$ is denoted by $\Delta L_{\sigma}^{i}$. Then there exists an orthogonal matrix $U_{\sigma}$ with appropriate dimensions such that

$$
\begin{aligned}
U_{\sigma}^{\mathrm{T}} L_{\sigma} U_{\sigma} & =\operatorname{diag}\left\{L_{\sigma}^{1}, L_{\sigma}^{2}, \ldots, L_{\sigma}^{n_{\sigma}}\right\} \\
U_{\sigma}^{\mathrm{T}} \Delta L_{\sigma}(t) U_{\sigma} & =\operatorname{diag}\left\{\Delta L_{\sigma}^{1}(t), \Delta L_{\sigma}^{2}(t), \ldots, \Delta L_{\sigma}^{n_{\sigma}}(t)\right\} \\
\tilde{x}_{F}^{\mathrm{T}}(t) U_{\sigma} & =\left[\tilde{x}_{\sigma F}^{1 \mathrm{~T}}(t), \tilde{x}_{\sigma F}^{2} \mathrm{~T}(t), \ldots, \tilde{x}_{\sigma F}^{n_{\sigma} \mathrm{T}}(t)\right] .
\end{aligned}
$$

Assumption 10. There exists a connectivity subset for MASs in each nonoverlapping time interval $\left[t_{r, j}, t_{r, s+1}\right) \subset\left[t_{r}, t_{r+1}\right), s=$ $1,2, \ldots, m_{r}$. For each follower, there exists at least one leader that has a path to the follower in the connectivity subset.

Considering the second-order MASs in the presence of dynamically switching and uncertain topologies with timevarying delays, dynamics (13) can be written as

$$
\dot{\tilde{x}}_{F}(t)=-H_{\sigma 2} \tilde{x}_{F}(t-\tau(t)),
$$

where $H_{\sigma 2}=\left[\begin{array}{cc}0_{n \times n} & -I_{n} \\ E_{\sigma F} & k_{1} I_{n}\end{array}\right], E_{\sigma F}=L_{\sigma F}+\Delta L_{\sigma F}(t)$.

In each subinterval $\left[t_{r, s}, t_{r, s+1}\right)$, dynamics (17) can be transformed into the following subsystems:

$$
\dot{\tilde{x}}_{\sigma F}^{i}(t)=-H_{\sigma 2}^{i} \widetilde{x}_{\sigma F}^{i}(t-\tau(t)), \quad i=1,2, \ldots, n_{\sigma},
$$

where $\tilde{x}_{\sigma F}^{i}(t)=x_{\sigma F}^{i}(t)+H_{\sigma 2}^{i}{ }^{-1} H_{\sigma 3}^{i} x_{\sigma L}^{i}(t), H_{\sigma 2}^{i}=$ $\left[\begin{array}{cc}0_{d_{\sigma F}^{i} \times d_{\sigma F}^{i}} & -I_{d_{\sigma F}^{i}} \\ E_{\sigma F}^{i} & k_{1} I_{d_{\sigma F}^{i}}^{i}\end{array}\right], H_{\sigma 3}^{i}=\left[\begin{array}{cc}0_{d_{\sigma F}^{i} \times d_{\sigma \Re}^{i}} & 0_{d_{\sigma F}^{i} \times d_{\sigma \Re}^{i}} \\ E_{\sigma F \Re}^{i} & 0_{d_{\sigma F}^{i} \times d_{\sigma \Re}^{i}}\end{array}\right], E_{\sigma F}^{i}=L_{\sigma F}^{i}+$ $\Delta L_{\sigma F}^{i}(t), E_{\sigma F \Re}^{i}=L_{\sigma F \Re}^{i}+\Delta L_{\sigma F \Re}^{i}(t), x_{\sigma F}^{i}(t)=\left[q_{\sigma F}^{i}{ }^{\mathrm{T}}(t)\right.$, $\left.p_{\sigma F}^{i \mathrm{~T}}(t)\right]^{\mathrm{T}}, x_{\sigma L}^{i \mathrm{~T}}(t)=\left[q_{\sigma L}^{i \mathrm{~T}}(t), p_{\sigma L}^{i \mathrm{~T}}(t)\right]^{\mathrm{T}}, q_{\sigma F}^{i}(t)=\left[q_{\sigma 1}^{i}(t), \ldots\right.$, $\left.q_{\sigma d_{\sigma F}^{i}}^{i}(t)\right]^{\mathrm{T}}, q_{\sigma L}^{i}(t)=\left[q_{\sigma d_{\sigma F}^{i}+1}^{i}(t), \ldots, q_{\sigma d_{\sigma}^{i}}^{i}(t)\right]^{\mathrm{T}}, p_{\sigma F}^{i}(t)=$ $\left[p_{\sigma 1}^{i}(t), \ldots, p_{\sigma d_{\sigma F}^{i}}^{i}(t)\right]^{\mathrm{T}}$, and $p_{\sigma L}^{i}(t)=\left[p_{\sigma d_{\sigma F}^{i}+1}^{i}(t), \ldots\right.$, $\left.p_{\sigma d_{\sigma}^{i}}^{i}(t)\right]^{\mathrm{T}}, i=1,2, \ldots, n_{\sigma}$.

Now, we consider a second-order MAS with uncertain topologies and communication time-varying delays. The stability and the convergence of MASs can be achieved as shown in the following Theorem 11.

Theorem 11. Consider a second-order dynamic system with dynamics (5) of $n$ followers and $m$ stationary leaders with the switching topologies, and suppose Assumptions 6, 7, and 10 hold. Control protocol (6) can solve the containment control of second-order MASs in the presence of uncertain topologies with time-varying delays, for each subinterval $\left[t_{r, s}, t_{r, s+1}\right)$, if there exists a constant $\varepsilon>0$ and an appropriate constant $\alpha>0$, such that

$$
\Pi_{\sigma}^{i}=\left[\begin{array}{ll}
\Pi_{11} & \Pi_{12} \\
\Pi_{12}^{\mathrm{T}} & \Pi_{22}
\end{array}\right]<0, \quad i=1,2, \ldots, n_{\sigma}
$$

where $\Pi_{11}=\left(\varepsilon-h^{-1}\right) I_{2 \times d_{\sigma F}^{i}}, \Pi_{12}=h^{-1} I_{2 \times d_{\sigma F}^{i}}+(\varepsilon h-1) \bar{H}_{\sigma 2}^{i}$, and $\Pi_{22}=\left(\varepsilon^{-1}-h^{-1}\right) I_{2 \times d_{\sigma F}^{i}}+\left(h+\varepsilon h^{2}\right) \bar{H}_{\sigma 2}^{i} \bar{H}_{\sigma 2}^{i}+h \alpha^{2} J_{\sigma}^{i}$, $\bar{H}_{\sigma 2}^{i}=\left[\begin{array}{cc}0_{d_{\sigma F}^{i} \times d_{\sigma F}^{i}} & -I_{d_{\sigma F}^{i}} \\ L_{\sigma F}^{i} & k_{1} I_{d_{\sigma F}^{i}}\end{array}\right], J_{\sigma}^{i}=\left[\begin{array}{cc}0_{d_{\sigma F}^{i}} & 0_{d_{\sigma F}^{i}} \\ I_{d_{\sigma F}^{i}} & 0_{d_{\sigma F}^{i}}^{i}\end{array}\right]$.

Proof. For MASs (17), we choose a Lyapunov-Krasovskii function candidate as

$$
V(t)=\tilde{x}_{F}^{\mathrm{T}}(t) \widetilde{x}_{F}(t)+\int_{t-h}^{t}(s-t+h) \dot{\tilde{x}}_{F}^{\mathrm{T}}(s) \dot{\tilde{x}}_{F}(s) d s
$$

Evidently, $V(t)$ is essentially a distributed Lyapunov function. By (16), $V(t)$ can be rewritten as

$$
\begin{aligned}
& V(t)=\sum_{i=1}^{n_{\sigma}}\left[\tilde{x}_{\sigma F}^{i \mathrm{~T}}(t) \tilde{x}_{\sigma F}^{i}(t)\right. \\
& \left.\quad+\int_{t-h}^{t}(s-t+h) \dot{\tilde{x}}_{\sigma F}^{i \mathrm{~T}}(s) \dot{\tilde{x}}_{\sigma F}^{i}(s) d s\right] .
\end{aligned}
$$

Calculating the derivative of $V(t)$ along the trajectories of (18) yields

$$
\begin{aligned}
& \dot{V}(t)=\sum_{i=1}^{n_{\sigma}}\left\{\left[-H_{\sigma 2}^{i} \tilde{x}_{\sigma F}^{i}(t-\tau(t))\right]^{\mathrm{T}} \tilde{x}_{\sigma F}^{i}(t)\right. \\
& +\tilde{x}_{\sigma F}^{i}(t)\left[-H_{\sigma 2}^{i} \tilde{x}_{\sigma F}^{i}(t-\tau(t))\right] \\
& \left.+h \dot{\tilde{x}}_{\sigma F}^{i}(t) \dot{\tilde{x}}_{\sigma F}^{i}(t)-\int_{t-h}^{t} \dot{\tilde{x}}_{\sigma F}^{i}(s) \dot{\tilde{x}}_{\sigma F}^{i}(s) d s\right\} .
\end{aligned}
$$

By Assumption 6 and Lemma 3, we can get $-\int_{t-h}^{t} \dot{\dot{x}}_{\sigma F}^{i}(s) \dot{\tilde{x}}_{\sigma F}^{i}(s) d s \leq-\int_{t-\tau(t)}^{t} \dot{\tilde{x}}_{\sigma F}^{i}(s) \dot{\vec{x}}_{\sigma F}^{i}(s) d s$ and $-\int_{t-\tau(t)}^{t} \dot{\tilde{x}}_{\sigma F}^{i \mathrm{~T}}(s) \dot{\vec{x}}_{\sigma F}^{i}(s) d s \leq-h^{-1}\left[\widetilde{x}_{\sigma F}^{i}(t)-\tilde{x}_{\sigma F}^{i}(t-\right.$ $\tau(t))]^{\mathrm{T}}\left[\tilde{x}_{\sigma F}^{i}(t)-\tilde{x}_{\sigma F}^{i}(t-\tau(t))\right]$. 


$$
\begin{aligned}
& \text { Denote } y_{\sigma}^{i}=\left[\widetilde{x}_{\sigma F}^{i}{ }^{\mathrm{T}}(t), \tilde{x}_{\sigma F}^{i}{ }^{\mathrm{T}}(t-\tau(t))\right]^{\mathrm{T}} \text {. It follows that } \\
& \dot{V}(t) \leq \sum_{i=1}^{n_{\sigma}}\left\{-\tilde{x}_{\sigma F}^{i}{ }^{\mathrm{T}}(t) H_{\sigma 2}^{i} \tilde{x}_{\sigma F}^{i}(t-\tau(t))\right. \\
& -\tilde{x}_{\sigma F}^{i \mathrm{~T}}(t-\tau(t)) H_{\sigma 2}^{i}{ }^{\mathrm{T}} \tilde{x}_{\sigma F}^{i}(t)+h \tilde{x}_{\sigma F}^{i \mathrm{~T}}(t-\tau(t)) \\
& \cdot H_{\sigma 2}^{i}{ }^{\mathrm{T}} H_{\sigma 2}^{i} \tilde{x}_{\sigma F}^{i}(t-\tau(t)) \\
& -h^{-1}\left[\widetilde{x}_{\sigma F}^{i}(t)-\tilde{x}_{\sigma F}^{i}(t-\tau(t))\right]^{\mathrm{T}} \\
& \left.\cdot\left[\tilde{x}_{\sigma F}^{i}(t)-\tilde{x}_{\sigma F}^{i}(t-\tau(t))\right]\right\}=\sum_{i=1}^{n_{\sigma}} y_{\sigma}^{i \mathrm{~T}} \Omega_{\sigma}^{i} y_{\sigma}^{i},
\end{aligned}
$$

where $\Omega_{\sigma}^{i}=\left[\begin{array}{cc}-h^{-1} I_{2 \times d_{\sigma F}^{i}} & h^{-1} I_{2 \times d_{\sigma F}^{i}}-H_{\sigma 2}^{i} \\ h^{-1} I_{2 \times d_{\sigma F}^{i}}-H_{\sigma 2}^{i \mathrm{~T}} & -h^{-1} I_{2 \times d_{\sigma F}^{i}}+h H_{\sigma 2}^{i}{ }^{\mathrm{T}} H_{\sigma 2}^{i}\end{array}\right]$.

Denote $\bar{H}_{\sigma 2}^{i}=\left[\begin{array}{cc}0_{d_{\sigma F}^{i} \times d_{\sigma F}^{i}} & -I_{d_{\sigma F}^{i}} \\ L_{\sigma F}^{i} & k_{1} I_{d_{\sigma F}^{i}}^{i}\end{array}\right]$ and $B_{\sigma}^{i}=$ $\left[\begin{array}{cc}0_{d_{\sigma F}^{i} \times d_{\sigma F}^{i}} & 0_{d_{\sigma F}^{i} \times d_{\sigma F}^{i}} \\ \Delta L_{\sigma F}^{i}(t) & 0_{d_{\sigma F}^{i} \times d_{\sigma F}^{i}}^{i}\end{array}\right]$. Then we have $H_{\sigma 2}^{i}=\bar{H}_{\sigma 2}^{i}+B_{\sigma}^{i}$. Let $\Phi_{\sigma}^{i}=\left[\begin{array}{cc}-h^{-1} I_{2 \times d_{\sigma F}^{i}} & h^{-1} I_{2 \times d_{\sigma F}^{i}}-\bar{H}_{\sigma 2}^{i} \\ h^{-1} I_{2 \times d_{\sigma F}^{i}}-\bar{H}_{\sigma 2}^{i \mathrm{~T}} & -h^{-1} I_{2 \times d_{\sigma F}^{i}}+h \bar{H}_{\sigma 2}^{i}{ }^{\mathrm{T}} \bar{H}_{\sigma 2}^{i}+h B_{\sigma}^{i \mathrm{~T}} B_{\sigma}^{i}\end{array}\right]$ and $\Psi_{\sigma}^{i}=$ $\left[\begin{array}{cc}0 & B_{\sigma}^{i} \\ B_{\sigma}^{i}{ }^{\mathrm{T}} & h \vec{H}_{\sigma 2}^{i}{ }^{\mathrm{T}} B_{\sigma}^{i}+h B_{\sigma}^{i}{ }^{\mathrm{T}} \vec{H}_{\sigma 2}^{i}\end{array}\right]$; we have $\Omega_{\sigma}^{i}=\Phi_{\sigma}^{i}+\Psi_{\sigma}^{i}$. By Lemma 4, it follows that $\Omega_{\sigma}^{i}=\Phi_{\sigma}^{i}+\Psi_{\sigma}^{i}=\Phi_{\sigma}^{i}+$ $\left[\begin{array}{c}0 \\ I_{2 \times d_{\sigma F}^{i}}^{i}\end{array}\right] B_{\sigma}^{i^{\mathrm{T}}}\left[\begin{array}{ll}I_{2 \times d_{\sigma F}^{i}} & h \bar{H}_{\sigma 2}^{i}\end{array}\right]+\left[\begin{array}{ll}I_{2 \times d_{\sigma F}^{i}} & h \bar{H}_{\sigma 2}^{i}\end{array}\right]^{\mathrm{T}} B_{\sigma}^{i}\left[\begin{array}{c}0 \\ I_{2 \times d_{\sigma F}^{i}}\end{array}\right]^{\mathrm{T}}<$ 0 is equivalent to $\Omega_{\sigma}^{i}=\Phi_{\sigma}^{i}+\Xi_{\sigma}^{i}=\Phi_{\sigma}^{i}+$ $\varepsilon\left[\begin{array}{ll}I_{2 \times d_{\sigma F}^{i}} & h \bar{H}_{\sigma 2}^{i}\end{array}\right]^{\mathrm{T}}\left[\begin{array}{ll}I_{2 \times d_{\sigma F}^{i}} & h \bar{H}_{\sigma 2}^{i}\end{array}\right] \mathcal{\varepsilon}^{-1}\left[\begin{array}{c}0 \\ I_{2 \times d_{\sigma F}^{i}}\end{array}\right]\left[\begin{array}{c}0 \\ I_{2 \times d_{\sigma F}^{i}}\end{array}\right]^{\mathrm{T}}<0$, where $\Xi_{\sigma}^{i}=\left[\begin{array}{cc}\varepsilon I_{2 \times d_{\sigma F}^{i}} & \varepsilon h \bar{H}_{\sigma 2}^{i} \\ \varepsilon h \bar{H}_{\sigma 2}^{i \mathrm{~T}} & \varepsilon h^{2} \bar{H}_{\sigma 2}^{i \mathrm{~T}} \bar{H}_{\sigma 2}^{i}{ }^{-1} I_{2 \times d_{\sigma F}^{i}}\end{array}\right], \varepsilon>0 . \mathrm{By}$ (11), we have $\Delta L_{\sigma F}^{i}{ }^{\mathrm{T}}(t) \Delta L_{\sigma F}^{i}(t) \leq \alpha^{2} I_{d_{\sigma F}^{i}}$ and $B_{\sigma}^{i^{\mathrm{T}}} B_{\sigma}^{i} \leq$ $\left[\begin{array}{cc}0_{d_{\sigma F}^{i} \times d_{\sigma F}^{i}} & 0_{d_{\sigma F}^{i} \times d_{\sigma F}^{i}} \\ \alpha^{2} I_{d_{\sigma F}^{i}} & 0_{d_{\sigma F}^{i} \times d_{\sigma F}^{i}}^{i}\end{array}\right]$. Then $\Phi_{\sigma}^{i} \leq\left[\begin{array}{ll}\Gamma_{11} & \Gamma_{12} \\ \Gamma_{12}^{\mathrm{T}} & \Gamma_{22}\end{array}\right]$, where $\Gamma_{11}=$ $-h^{-1} I_{2 \times d_{\sigma F}^{i}}, \Gamma_{12}=h^{-1} I_{2 \times d_{\sigma F}^{i}}-\bar{H}_{\sigma 2}^{i}$, and $\Gamma_{22}=-h^{-1} I_{2 \times d_{\sigma F}^{i}}+$ $h \bar{H}_{\sigma 2}^{i}{ }^{\mathrm{T}} \bar{H}_{\sigma 2}^{i}+h \alpha^{2} J_{\sigma}^{i}, J_{\sigma}^{i}=\left[\begin{array}{cc}0_{d_{\sigma F}^{i}} & 0_{d_{\sigma F}^{i}} \\ I_{d_{\sigma F}^{i}} & 0_{d_{\sigma F}^{i}}^{i}\end{array}\right]$. Therefore, $\Omega_{\sigma}^{i}$ is negative definite if condition (19) is satisfied. Thus $\dot{V}(t)<0$ and the stability of the dynamically switching system (17) is guaranteed.

Now we know system (17) is asymptotically stable. So $\tilde{x}_{F}(t)$ and $\tilde{x}_{F}(t-\tau(t))$ are bounded, and $\dot{\tilde{x}}_{F}(t)$ is also bounded by (13). Consequently the derivative of $\sum_{i=1}^{n_{\sigma}} \tilde{y}_{\sigma}^{i \mathrm{~T}}\left[\Omega_{\sigma}^{i}+\Psi_{\sigma}^{i}\right] \widetilde{y}_{\sigma}^{i}$ is bounded by (16) and (18). It follows that $\int_{0}^{+\infty}\left(\sum_{i=1}^{n_{\sigma}} y_{\sigma}^{i}(s)^{\mathrm{T}} \Omega_{\sigma}^{i} y_{\sigma}^{i}(s)\right) d s$ exists and is finite since $\sum_{i=1}^{n_{\sigma}} y_{\sigma}^{i \mathrm{~T}} \Omega_{\sigma}^{i} y_{\sigma}^{i}<0$ and $0 \geq \int_{0}^{t} \sum_{i=1}^{n_{\sigma}} y_{\sigma}^{i}(s)^{\mathrm{T}} \Omega_{\sigma}^{i} y_{\sigma}^{i}(s) d s \geq$ $\int_{0}^{t} \dot{V}(s) d s=V(t)-V(0)$. By Lemma 5, we can get $\lim _{t \rightarrow+\infty} \sum_{i=1}^{n_{\sigma}} y_{\sigma}^{i \mathrm{~T}} \Omega_{\sigma}^{i} y_{\sigma}^{i}=0$ and $\lim _{t \rightarrow+\infty} y_{\sigma}^{i}=0$ since $\Omega_{\sigma}^{i}<0$. Then $\lim _{t \rightarrow+\infty} \tilde{x}_{\sigma F}^{i}(t)=\tilde{x}_{\sigma F}^{i}(t-\tau(t))=0$. Thus $\lim _{t \rightarrow+\infty} x_{\sigma F}^{i}(t)=-H_{\sigma 2}^{i}{ }^{-1} H_{\sigma 3}^{i} x_{\sigma L}^{i}(t)$.

Furthermore, in any subinterval $\left[t_{r, s}, t_{r, s+1}\right) \subset\left[t_{r}, t_{r+1}\right)$, $s=1,2, \ldots, m_{r}, \lim _{t \rightarrow+\infty} x_{\sigma F}^{i}(t)=-H_{\sigma 2}^{i}{ }^{-1} H_{\sigma 3}^{i} x_{\sigma L}^{i}(t)$ and $\lim _{t \rightarrow+\infty} q_{\sigma F}^{i}(t)=-E_{\sigma F}^{i}{ }^{-1} E_{\sigma F \Re}^{i} q_{\sigma L}^{i}(t), i=1,2, \ldots, n_{\sigma}$. Therefore, in the connected portion of MASs in the subinterval $\left[t_{r, s}, t_{r, s+1}\right)$ and $\left[t_{r, s+1}, t_{r, s+2}\right)$, that is, in the subinterval $\left[t_{r, s}, t_{r, s+2}\right), \lim _{t \rightarrow+\infty} x_{\sigma F}^{i \prime}(t)=-H_{\sigma 2}^{i}{ }^{-1 \prime} H_{\sigma 3}^{i \prime} x_{\sigma L}^{i \prime}(t)$ and $\lim _{t \rightarrow+\infty} q_{\sigma F}^{i \prime}(t)=-E_{\sigma F}^{i}{ }^{-1 \prime} E_{\sigma F \Re}^{i \prime} q_{\sigma L}^{i \prime}(t)$ still hold, $i=$ $1,2, \ldots, n_{\sigma}^{\prime}$. Then by induction, according to Assumption 7 , $\lim _{t \rightarrow+\infty} x_{F}(t)=-H_{2}{ }^{-1} H_{3} x_{L}(t)$ and $\lim _{t \rightarrow+\infty} q_{F}(t)=$ $-E_{F}^{-1} E_{F L} q_{L}(t)$, since all agents are jointly connected in each $\left[t_{r}, t_{r+1}\right)$. Then we can conclude that the containment control of the second-order MASs with time-varying delays and uncertain topologies can be realized by Lemma 9 .

Corollary 12. Consider the second-order MASs with dynamics (5) and suppose Assumptions 6, 7, and 10 hold, for the case where the communication delay $\tau(t)$ is time invariant; that is, $\tau(t)=\tau$ is a constant in (6). Control protocol (6) can solve the containment control of second-order MASs in the presence of uncertain topologies with communication delays, for each subinterval $\left[t_{r, s}, t_{r, s+1}\right)$, if there exists a constant $\varepsilon>0$ and an appropriate constant $\alpha>0$, such that

$$
\Lambda_{\sigma}^{i}=\left[\begin{array}{cc}
\Lambda_{11} & \Lambda_{12} \\
* & \Lambda_{22}
\end{array}\right]<0, \quad i=1,2, \ldots, n_{\sigma}
$$

where $\Lambda_{11}=(\varepsilon-1) I_{2 \times d_{\sigma F}^{i}}, \Lambda_{12}=(\varepsilon \tau-1) \bar{H}_{\sigma 2}^{i}$, and $\Lambda_{22}=$ $\left(\varepsilon^{-1}-1\right) I_{2 \times d_{\sigma F}^{i}}+\left(\tau+\varepsilon \tau^{2}\right) \bar{H}_{\sigma 2}^{i} \bar{H}_{\sigma 2}^{i}+\tau \alpha^{2} J_{\sigma}^{i} ; \bar{H}_{\sigma 2}^{i}$ and $J_{\sigma}^{i}$ are the same as $\bar{H}_{\sigma 2}^{i}$ and $J_{\sigma}^{i}$ in (19).

\section{Simulations}

Consider dynamic switching topology with 5 followers and 3 leaders as shown in Figure 1. The followers' set and the leaders' set are denoted by $F=\{1,2,3,4,5\}$ and $\Re=\{6,7,8\}$, respectively. Suppose that the communication topology of the MASs randomly switches in $\{G 1, G 2, G 3\}$ at $t=k T, k=$ $0,1, \ldots, T=0.5 \mathrm{~s}$, and the connection weights of each edge are all set to 1 in this paper.

By the union topology of graphs $G 1 \sim G 3$, the system matrix $L_{\sigma F}$ can be obtained. Solving (19) by the Matlab's LMI toolbox, we can get $k_{1}>4.3278$ and $h<0.4621$. Let $k_{1}=$ 4.4 and $\Delta L_{\sigma F}=\alpha \sin (t) * L_{\sigma F}$ with $\alpha=0.1$. The timevarying delays of MASs are taken as $\tau(t)=0.2+0.2 \sin t$ in the simulations. For the MASs with multiple stationary leaders, we consider five agents are guided by three leaders moving in a plane. The initial position of followers and leaders are chosen as $q_{1}(0)=(1,1)^{\mathrm{T}}, q_{2}(0)=(1,3)^{\mathrm{T}}, q_{3}(0)=$ $(3,1)^{\mathrm{T}}, q_{4}(0)=(3,5)^{\mathrm{T}}, q_{5}(0)=(5,3)^{\mathrm{T}}, q_{6}(0)=(7,10)^{\mathrm{T}}$, $q_{7}(0)=(10,7)^{\mathrm{T}}$, and $q_{8}(0)=(10,10)^{\mathrm{T}}$. The initial velocities of followers and leaders are chosen as $p_{1}(0)=(1,1)^{\mathrm{T}}, p_{2}(0)=$ $(2,2)^{\mathrm{T}}, p_{3}(0)=(1,1)^{\mathrm{T}}, p_{4}(0)=(2,2)^{\mathrm{T}}, p_{5}(0)=(3,3)^{\mathrm{T}}$, and $p_{6}(0)=p_{7}(0)=p_{8}(0)=0$. Simulation results are shown in Figure 2, which shows that those followers can asymptotically converge to the triangle formed by three leaders; that is, containment control of second-order MASs in the presence of uncertain topologies with multiple stationary leaders and time-varying delays can be achieved. 


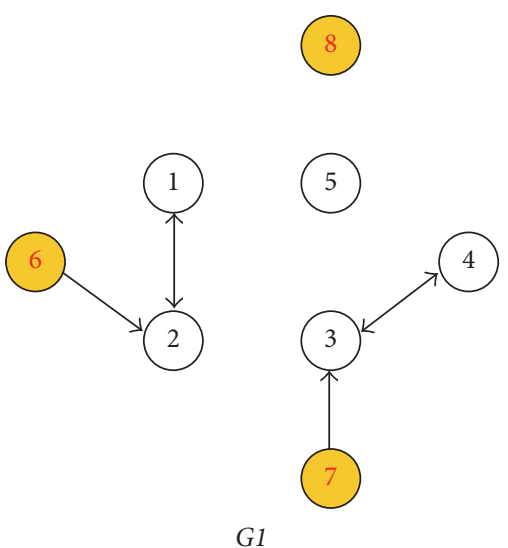

(a)

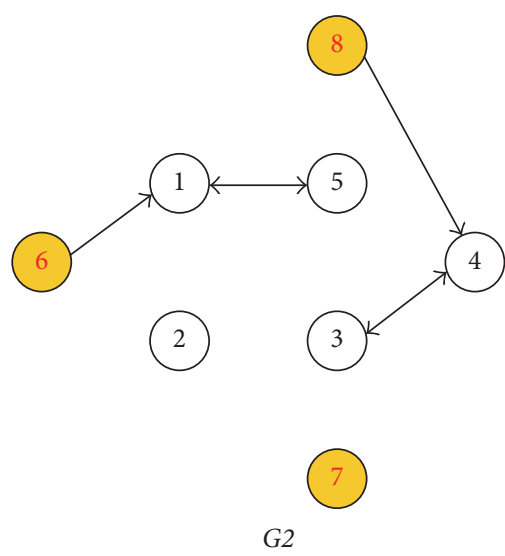

(b)

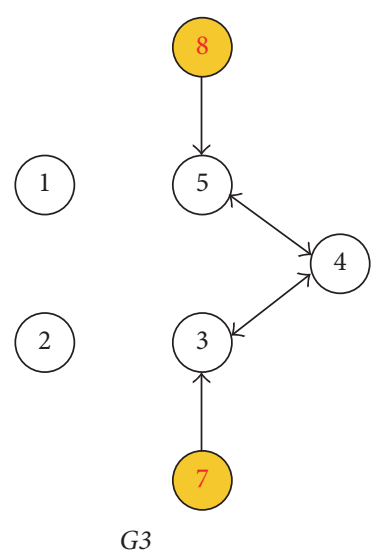

(c)

FIgURE 1: Three possible graphs of the communication topology.

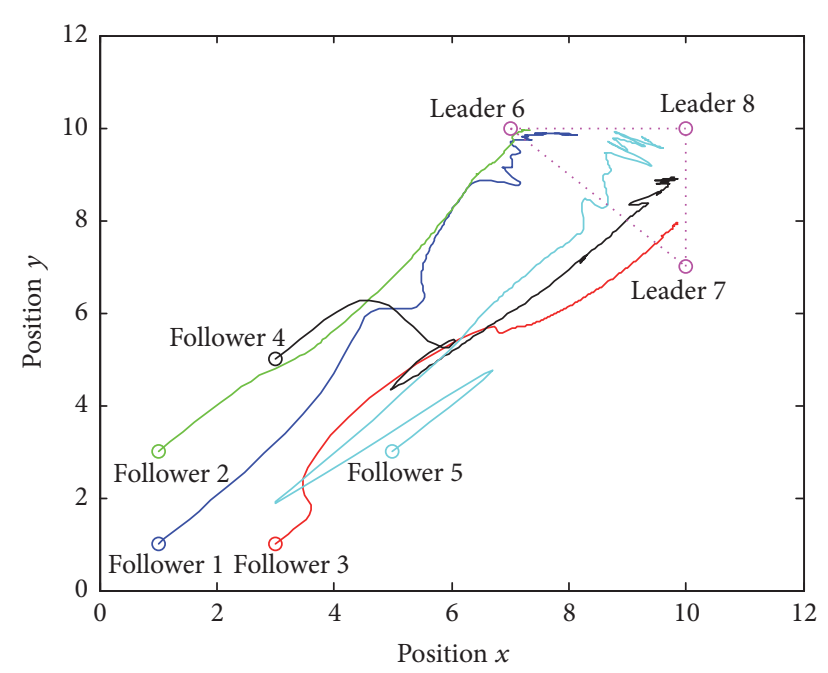

FIGURE 2: State trajectories of MASs with uncertain topologies.

\section{Conclusion}

In this paper, the distributed containment control for secondorder MASs with multiple stationary leaders and uncertain topologies is investigated. The control algorithm of MASs with time-varying delays and jointly connected topologies is proposed. By applying modern control theory and algebraic graph theory, the convergence of MASs for the proposed containment control algorithm is analyzed via LyapunovKrasovskii method. Some sufficient conditions in terms of linear matrix inequalities (LMIs) are given for containment control. The correctness and effectiveness of our theoretical results for uncertain MASs with time-varying delays are demonstrated by some simulation examples. The containment control problem for nonlinear systems will be our future work.

\section{Competing Interests}

The authors declare that they have no competing interests.

\section{Acknowledgments}

This research is supported by the National Natural Science Foundation of China (Grant nos. 61573200, 61573199, 61273152, and 61673200) and the Tianjin Natural Science Foundation of China (Grant nos. 14JCYBJC18700 and 14JCZDJC39300).

\section{References}

[1] Z.-X. Liu and Z.-Q. Chen, "Discarded consensus of network of agents with state constraint," IEEE Transactions on Automatic Control, vol. 57, no. 11, pp. 2869-2874, 2012.

[2] H.-Y. Yang, L. Guo, and H.-L. Zou, "Robust consensus of multiagent systems with time-delays and exogenous disturbances," International Journal of Control, Automation and Systems, vol. 10, no. 4, pp. 797-805, 2012.

[3] Y. Liu, H. Min, S. Wang, Z. Liu, and S. Liao, "Distributed adaptive consensus for multiple mechanical systems with switching topologies and time-varying delay," Systems \& Control Letters, vol. 64, no. 1, pp. 119-126, 2014.

[4] M. Diao, Z. Duan, and G. Wen, "Consensus tracking of linear multi-agent systems under networked observability conditions," International Journal of Control, vol. 87, no. 8, pp. 14781486, 2014.

[5] S. Su and Z. Lin, "Distributed consensus control of multi-agent systems with higher order agent dynamics and dynamically changing directed interaction topologies," Institute of Electrical and Electronics Engineers. Transactions on Automatic Control, vol. 61, no. 2, pp. 515-519, 2016.

[6] G. Notarstefano, M. Egerstedt, and M. Haque, "Containment in leader-follower networks with switching communication topologies," Automatica, vol. 47, no. 5, pp. 1035-1040, 2011.

[7] Y. Cao, W. Ren, and M. Egerstedt, "Distributed containment control with multiple stationary or dynamic leaders in fixed and switching directed networks," Automatica, vol. 48, no. 8, pp. 1586-1597, 2012.

[8] Z. Li, W. Ren, X. Liu, and M. Fu, "Distributed containment control of multi-agent systems with general linear dynamics in the presence of multiple leaders," International Journal of Robust and Nonlinear Control, vol. 23, no. 5, pp. 534-547, 2013. 
[9] Z. Liu, Q. Jin, and Z. Chen, "Distributed containment control for bounded unknown second-order nonlinear multi-agent systems with dynamic leaders," Neurocomputing, vol. 168, pp. 1138-1143, 2015.

[10] Y. Sun, "Average consensus in networks of dynamic agents with uncertain topologies and time-varying delays," Journal of the Franklin Institute, vol. 349, no. 3, pp. 1061-1073, 2012.

[11] D. Han and G. Chesi, "Robust discrete-time consensus of multiagent systems with uncertain interaction," in Proceedings of the IEEE International Conference on Control Applications (CCA '12), pp. 1136-1141, October 2012.

[12] Y. Shang, "Average consensus in multi-agent systems with uncertain topologies and multiple time-varying delays," Linear Algebra and Its Applications, vol. 459, pp. 411-429, 2014.

[13] Y. Shang, "Consensus seeking over Markovian switching networks with time-varying delays and uncertain topologies," Applied Mathematics and Computation, vol. 273, pp. 1234-1245, 2016.

[14] H. J. Savino, C. R. P. dos Santos, F. O. Souza, L. C. A. Pimenta, M. de Oliveira, and R. M. Palhares, "Conditions for consensus of multi-agent systems with time-delays and uncertain switching topology," IEEE Transactions on Industrial Electronics, vol. 63, no. 2, pp. 1258-1267, 2016.

[15] Q. Ma and G. Miao, "Distributed containment control of linear multi-agent systems," Neurocomputing, vol. 133, pp. 399-403, 2014.

[16] F.-Y. Wang, H.-Y. Yang, and F.-J. Han, "Flocking motion of dynamic networked systems with multiple leaders," Acta Electronica Sinica, vol. 44, no. 7, pp. 1751-1756, 2016.

[17] K. Liu, G. Xie, and L. Wang, "Containment control for secondorder multi-agent systems with time-varying delays," Systems \& Control Letters, vol. 67, no. 1, pp. 24-31, 2014.

[18] F. Y. Wang, H. Y. Yang, and F. J. Han, "Containment control of second-order multi-agent systems with jointly-connected topologies and varying delays," in Proceedings of the 2015 Chinese Intelligent Systems Conference, vol. 2 of Lecture Notes in Electrical Engineering, pp. 45-53, Springer, Berlin, Germany, 2016.

[19] J. Chen, Z.-H. Guan, C. Yang, T. Li, D.-X. He, and X.-H. Zhang, "Distributed containment control of fractional-order uncertain multi-agent systems," Journal of the Franklin Institute, vol. 353, no. 7, pp. 1672-1688, 2016.

[20] F. Wang, H. Yang, S. Zhang, and F. Han, "Containment control for first-order multi-agent systems with time-varying delays and uncertain topologies," Communications in Theoretical Physics, vol. 66, no. 2, pp. 249-255, 2016.

[21] P. Lin and Y. Jia, "Consensus of a class of second-order multiagent systems with time-delay and jointly-connected topologies," IEEE Transactions on Automatic Control, vol. 55, no. 3, pp. 778-784, 2010.

[22] Y. Cao, D. Stuart, W. Ren, and Z. Meng, "Distributed containment control for multiple autonomous vehicles with doubleintegrator dynamics: algorithms and experiments," IEEE Transactions on Control Systems Technology, vol. 19, no. 4, pp. 929938, 2011.

[23] Z. Meng, W. Ren, and Z. You, "Distributed finite-time attitude containment control for multiple rigid bodies," Automatica, vol. 46, no. 12, pp. 2092-2099, 2010.

[24] Y. Cao and W. Ren, "Containment control with multiple stationary or dynamic leaders under a directed interaction graph," in Proceedings of the 48th IEEE Conference on Decision and
Control, 2009 Held Jointly with the 2009 28th Chinese Control Conference (CDC/CCC '09), pp. 3014-3019, Shanghai, China, December 2009.

[25] Y. Gu, S. Wang, Q. Li, Z. Cheng, and J. Qian, "On delaydependent stability and decay estimate for uncertain systems with time-varying delay," Automatica, vol. 34, no. 8, pp. 10351039, 1998.

[26] I. R. Petersen and C. V. Hollot, "A Riccati equation approach to the stabilization of uncertain linear systems," Automatica, vol. 22, no. 4, pp. 397-411, 1986.

[27] P. Lin, K. Qin, H. Zhao, and M. Sun, "A new approach to average consensus problems with multiple time-delays and jointlyconnected topologies," Journal of the Franklin Institute, vol. 349, no. 1, pp. 293-304, 2012. 


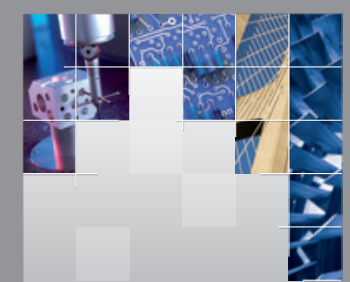

\section{Enfincering}
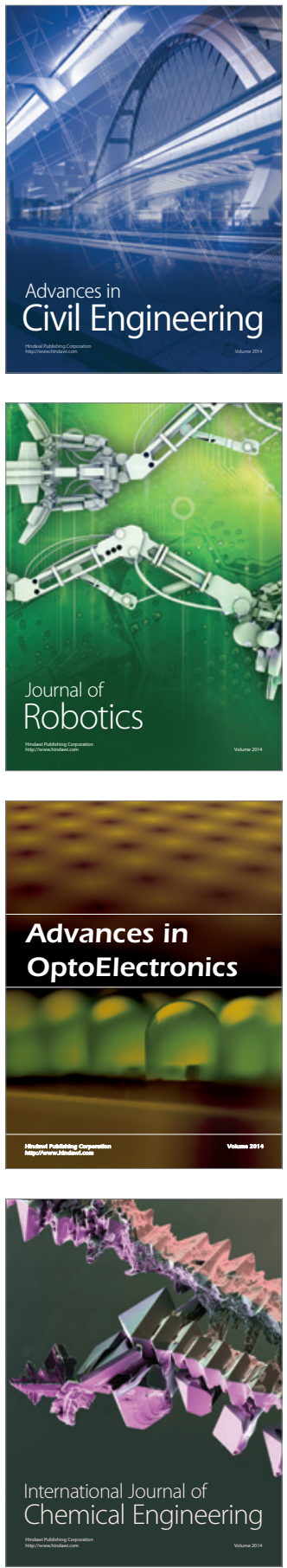

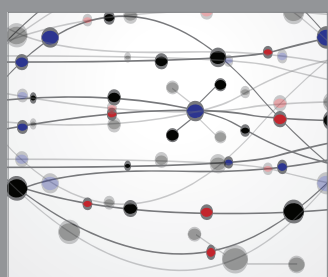

The Scientific World Journal

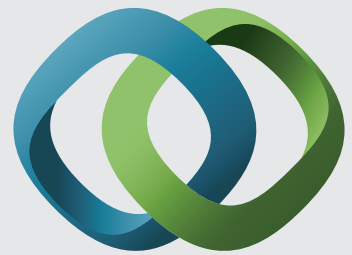

\section{Hindawi}

Submit your manuscripts at

https://www.hindawi.com
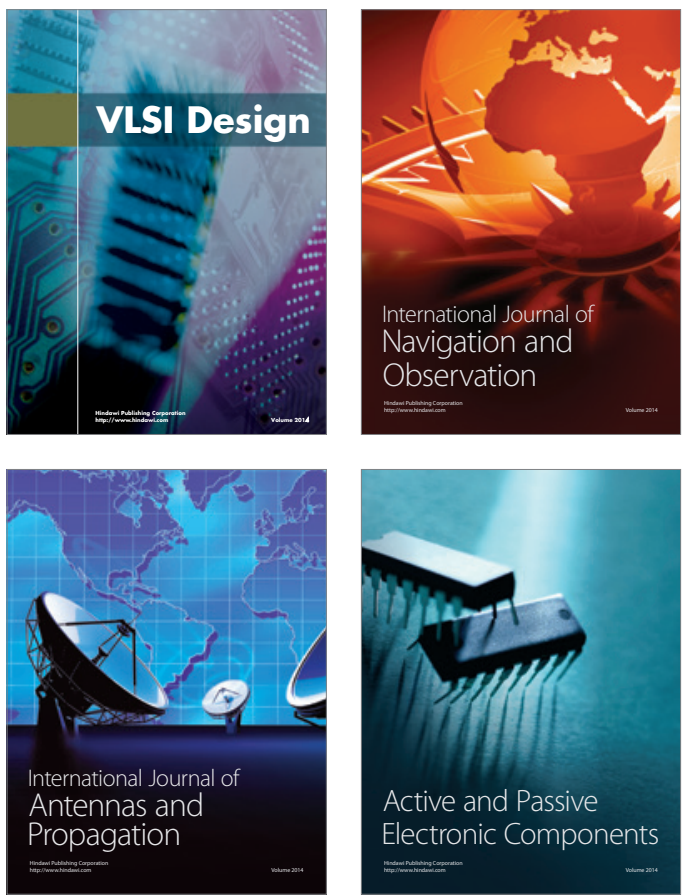
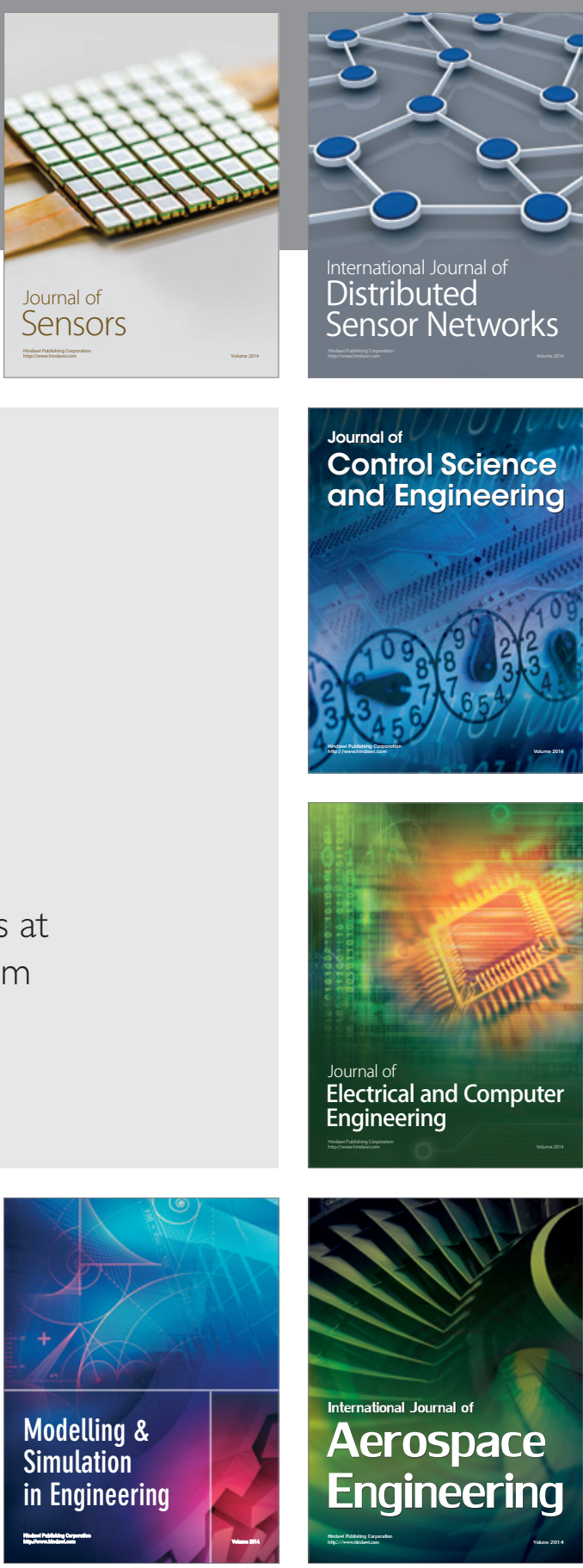

International Journal of

Distributed

Sensor Networks

$-$

Joumal of

Control Science

and Engineering
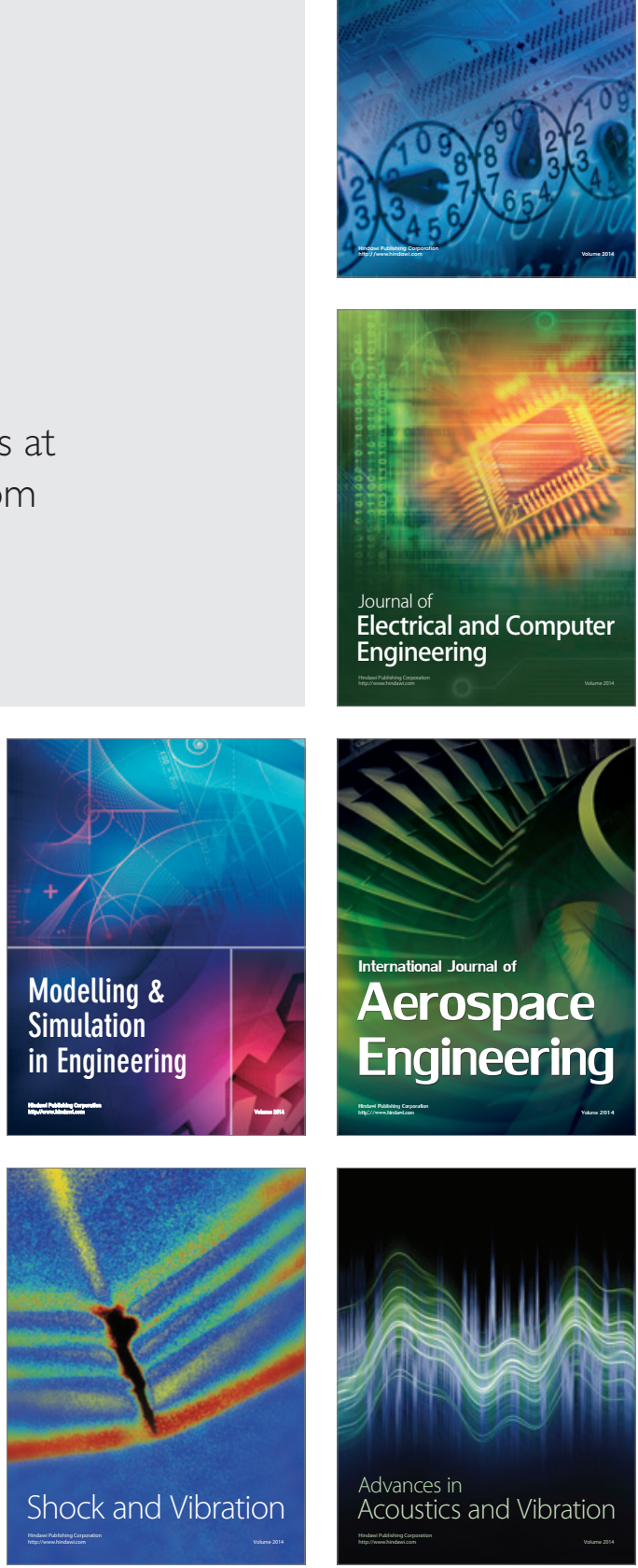